\title{
Sistema adaptativo de inferencia neuro-difusa (ANFIS) para la estimación de la radiación solar global
}

INVESTIGACIÓN

e INNOVACIÓN en INGENERÍAS ISSN2344-8652

Recibido: 18/08/2020

Aceptado: 11/11/2020

Publicado: 04/01/2021

Correspondencia de autores: dianacarolinabgbag@ufps.edu.co

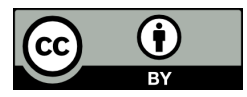

Copyrith 2020

by Investigación e Innovación en Ingenierias

\section{Adaptive Neuro-Fuzzy Inference System (ANFIS) for the estimation of global solar radiation}

\author{
Diana Carolina Bastos Guerrero iD Mario Joaquín Illera Bustos iD \\ Sergio Basilio Sepúlveda Mora \\ Universidad Francisco de Paula Santander
}

Resumen

Objetivo: Ajustar un sistema adaptativo de inferencia neuro-difusa (ANFIS) para la estimación de la radiación solar global en la ciudad de Cúcuta. Metodología: A partir de registros históricos del Instituto de Hidrología, Meteorología y Estudios Ambientales (IDEAM) se obtuvieron las variables: temperatura ambiente, brillo solar, velocidad del viento, humedad relativa, hora de medición y radiación solar global entre los años 2005 y 2015. Usando la herramienta Fuzzy Logic Toolbox de Matlab y los datos del IDEAM se crearon redes ANFIS con diferentes configuraciones. Después de comparar los errores estadísticos, se escogió el modelo ANFIS que permitió minimizar el error cuadrático medio (RMSE). Resultados: Los errores estadísticos del modelo son: R2 $=0.9115, \mathrm{RMSE}=124,23 \mathrm{Wh} / \mathrm{m} 2$ y $\mathrm{MAPE}=27,8$ $\%$, evidenciando una alta precisión para la estimación de la radiación solar global en el territorio seleccionado. Conclusiones: La red ANFIS propuesta es un modelo basado en inteligencia artificial con precisión suficiente para ser usado en el diseño de sistemas fotovoltaicos en la región, que no cuenta con una amplia red de piranómetros para medir el recurso solar.

Palabras clave: ANFIS, modelo de estimación indirecto, radiación solar global.

Abstract

Objective: Adjust an adaptive neuro-diffuse inference system (ANFIS) for the estimation of global solar radiation in the city of Cúcuta. Methodology: From historical records of the Institute of Hydrology, Meteorology and Environmental Studies (IDEAM) the variables were obtained: ambient temperature, solar brightness, wind speed, relative humidity, measurement time and global solar radiation between the years 2005 and 2015. Using The Fuzzy Logic Toolbox from Matlab and the IDEAM data were created ANFIS networks with different configurations. After comparing the statistical errors the ANFIS model was chosen, which allowed minimizing the mean square error (RMSE). Results: The statistical errors of the model are: $\mathrm{R} 2=0.9115, \mathrm{RMSE}=124.23 \mathrm{Wh} / \mathrm{m} 2$ and $\mathrm{MAPE}=27.8 \%$, showing high precision for estimating global solar radiation in the selected territory. Conclusions: The proposed ANFIS network is a model based on artificial intelligence with sufficient precision to be used in the design of photovoltaic systems in the region, which does not have a wide network of pyranometers to measure the solar resource.

Keywords: ANFIS, indirect estimation model, global solar radiation. 


\section{Introducción}

El uso de la energía eléctrica se convirtió en un factor fundamental para el desarrollo de la humanidad. Durante los últimos años, las principales técnicas para la producción de energía eléctrica provienen de fuentes de energía convencional o no renovable; esto trae consigo el aumento de la polución, relacionado directamente con la generación de gases que incrementan el efecto invernadero. Con base en la situación ambiental expuesta, se ha considerado brindar un rol más significativo a la generación de energía eléctrica a partir de fuentes renovables no convencionales, debido a que son fuentes virtualmente inagotables y que en su mayoría cumplen un papel responsable con el medio ambiente [1]. Por lo tanto, la cuantificación de los recursos renovables en una región particular es crucial para la transición a este tipo de fuentes de energía. Por ejemplo, Henao, Báez y Pedroza exploraron el potencial eólico en la ciudad de Cúcuta, Colombia, pero concluyeron que la velocidad del viento promedio no es suficiente para que sea una alternativa económicamente viable [2].

Considerando lo anterior, la energía solar fotovoltaica se ha convertido en una de las fuentes renovables más importantes del planeta, debido a la disminución de sus costos de producción y a su fácil implementación en zonas de difícil acceso donde no llega la red de energía eléctrica convencional [1]. De esta forma, es que el conocimiento de la disponibilidad de la radiación solar se ha convertido en un tema de estudio indispensable, ya que es uno de los factores más importantes para el correcto dimensionamiento de sistemas y tecnologías que transforman la energía solar, por ejemplo, en electricidad a través de los sistemas fotovoltaicos o en calor por medio de los sistemas térmicos [3]. En Colombia, el Instituto de Hidrología, Meteorología y Estudios Ambientales (IDEAM) ha desarrollado un mapa interactivo de la radiación solar en este país [4]; sin embargo, este mapa es generalizado y agrupa grandes extensiones geográficas con diferentes condiciones climáticas; por otra parte, los autores en [5] utilizaron datos históricos del IDEAM para caracterizar la radiación solar global en la ciudad de Cúcuta usando estadística descriptiva. No obstante, los datos del IDEAM para la ciudad de Cúcuta son muy limitados, ya que solo se dispone de información entre 2005 y 2015, la cual posee una gran cantidad de registros incompletos. Adicionalmente, Noriega et al. [6] desarrollaron un modelo basado en redes neuronales artificiales para estimar la radiación solar en la costa atlántica colombiana, y obtuvieron un coeficiente de correlación $\mathrm{R}=0.77$.

Los datos de radiación solar son obtenidos con mayor precisión a partir de instrumentos de medición como los piranómetros. Sin embargo, debido al alto costo de calibración y mantenimiento de estos equipos, los datos de radiación solar son limitados o escasos en muchas estaciones meteorológicas a nivel mundial [7]; en el caso específico de Colombia, de las 5447 estaciones instaladas en el país, aproximadamente el $2 \%$ miden datos de irradiación.

Por esta razón, estimar la radiación solar a partir de variables meteorológicas de amplia distribución y con facilidad de acceso a sus registros como la temperatura, humedad, entre otras, se ha convertido en un eje importante de investigación, teniendo en cuenta que se puede proporcionar información solar, en lugares donde no se registra o donde su registro está desactualizado, a partir de otras variables disponibles [8].

Los Sistemas Adaptativos de Inferencia Neuro-Difusa (ANFIS) han demostrado ser eficaces para la predicción de variables en entornos complejos [9]. Varios estudios recientes [7,10,11,12,13,14,15,16,17,18] han propuesto modelos ANFIS para la estimación de la radiación solar global a partir de otras variables meteorológicas de más fácil acceso, y concluyeron que el modelo debe ajustarse a las condiciones climáticas particulares de cada lugar y que ANFIS se desempeña mejor que los modelos empíricos y los modelos estadísticos. 
Por ejemplo, Olatomiwa et al. [7] consideraron la temperatura máxima, temperatura mínima y brillo solar, como las variables de entrada para su modelo ANFIS en Nigeria; esta investigación obtuvo como resultado un R2 = 0.854; por otra parte, Mohanty [18] determinó que el brillo solar, temperatura ambiente, humedad relativa y el índice de claridad serían las variables de entrada para su red de estimación; el propósito de esa investigación fue comparar los resultados del modelo ANFIS con los arrojados aplicando la ecuación de Angström-Prescott (modelo empírico de estimación).

Sin embargo, en algunos estudios [19,20] consideraron que la radiación solar global sería un factor importante tanto en la entrada de la red como en la salida. Sumithira y Nirmal [19] tomaron como variables de entrada la latitud y longitud, mientras que Mellit et al. [20] consideraron la temperatura ambiente y el brillo solar. Adicionalmente, estos dos estudios incluyeron como variable de entrada la radiación solar global para el entrenamiento y comprobación de la red. La inclusión de este parámetro como variable de entrada arrojó mejores resultados con un R2 = 0.9898 en el caso de Sumithira y Nirmal [19] y un R2 = 0.97 para Mellit et al. [20]. Si bien usar la radiación solar global como entrada del modelo mejora sustancialmente la precisión, este procedimiento no tendría aplicación en lugares donde la radiación solar global no es medida, y se desea estimar a partir de otras variables disponibles en ese lugar.

Así, la presente investigación tiene por objetivo la construcción de un modelo de estimación de la radiación solar global en la ciudad de Cúcuta, Norte de Santander, utilizando un sistema adaptativo de inferencia neuro difusa (ANFIS), como una herramienta adicional de información sobre el recurso solar en la región.

\section{Metodología}

Para el diseño de la red ANFIS se adquirieron datos históricos de humedad relativa, temperatura ambiente, brillo solar, velocidad del viento y radiación solar de una estación meteorológica del IDEAM ubicada en Cúcuta. Los datos fueron pre-procesados, normalizados y utilizados para entrenar 20 configuraciones distintas de redes ANFIS. Los parámetros que se consideraron para cada red fueron: el número de funciones de membresía (FMs), el tipo de FMs, y el algoritmo de aprendizaje. Posteriormente se escogió la red de mejor desempeño con base en los errores estadísticos y se calculó el error de comprobación (testing) con datos desconocidos por la red. A continuación, se describe con detalle cada etapa del proceso.

\section{Sistema de inferencia neuro-difusa (ANFIS)}

El modelo que se implementa en este trabajo es un modelo híbrido de inteligencia artificial el cual está comprendido por una red neuronal artificial con inferencia difusa. La idea principal del modelo consiste en que los nodos (puntos de procesamiento de información) de las diferentes capas de una red neuronal convencional, contienen parámetros difusos, siendo equivalente a los sistemas de inferencia difusa (FIS, por sus siglas en inglés) con parámetros distribuidos [21].

ANFIS sigue el patrón de una red adaptativa comprendida por nodos fijos y nodos ajustables. En la figura 1 se muestra una red de alimentación multicapa donde cada nodo realiza una función en específico (función del nodo) en las señales entrantes; además de los parámetros pertenecientes a cada nodo, la figura muestra la estructura de la red adaptativa con sus correspondientes capas. 
Figural. Estructura básica de una red ANFIS.

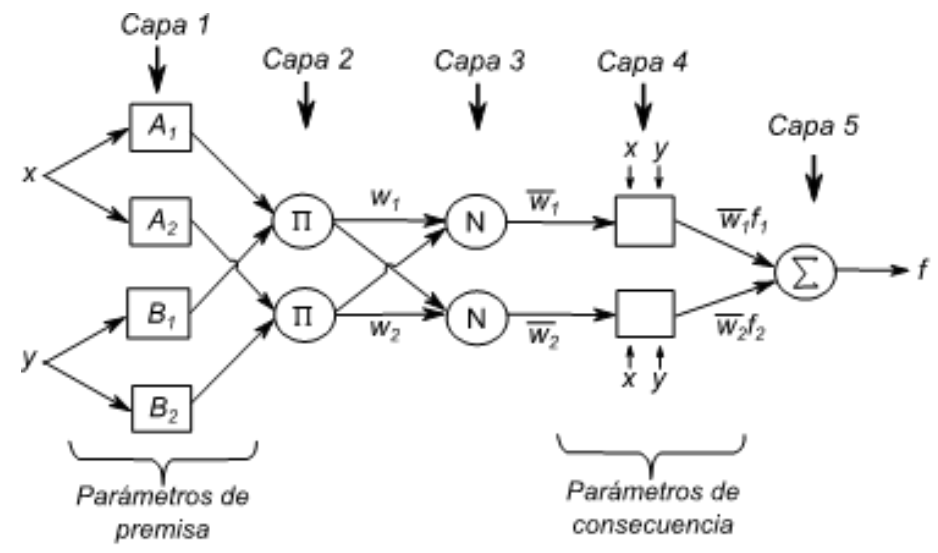

Fuente: Adaptada de [22]

El tipo de sistema neuro-difuso utilizado se basa en dividir la representación del conocimiento previo en subconjuntos para reducir el espacio de búsqueda y utiliza el algoritmo de propagación inversa para ajustar los parámetros difusos [23]. Estos subconjuntos son denominados Funciones de Membresía (FM). El sistema resultante (mostrado en la figura 2) es una red neuronal adaptativa funcionalmente equivalente a un sistema de inferencia Takagi-Sugeno de primer orden, donde la relación entrada-salida es lineal.

Figura 2. Razonamiento difuso de tipo Sugeno de primer orden.

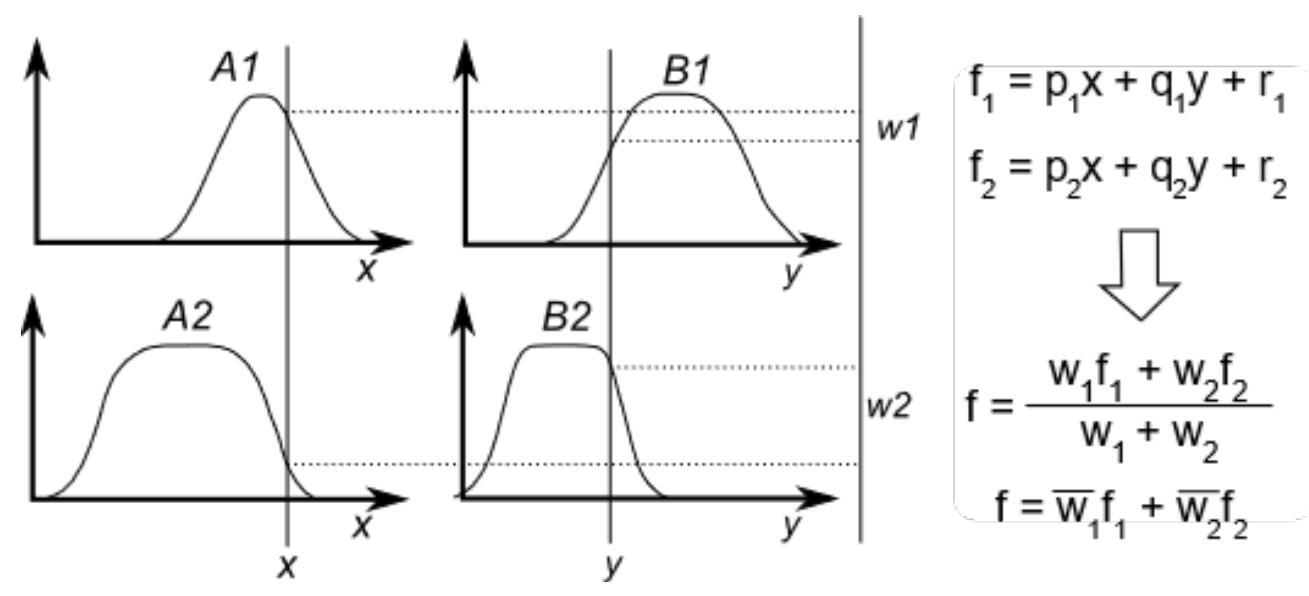

Fuente: Tomado de [21] 


\section{Acondicionamiento de los datos}

Para la obtención de la información se realizó una solicitud al IDEAM [24], con el fin de adquirir los registros de la base de datos de la estación meteorológica satelital ubicada en la Universidad Francisco de Paula Santander, en la ciudad de Cúcuta, Colombia. Las variables brindadas por el IDEAM son la humedad relativa, temperatura ambiente, brillo solar, velocidad del viento y radiación solar global. Cada conjunto de datos viene definido con el día, mes y año en el que se tomó la medición; adicionalmente, el IDEAM toma sus mediciones de manera horaria lo que significa que la base de datos también suministra la hora en que fue realizada la adquisición de la información.

Los datos solicitados están comprendidos en un periodo de 10 años (2005-2015); fueron eliminados los registros donde hacían falta por lo menos una de las 5 variables meteorológicas y donde sus variables se salían del rango de operación, obteniendo al final un total de 23.220 registros horarios.

Con la información anterior, se realiza un pre-procesado de los datos para garantizar su confiabilidad. El pre-procesamiento consiste en la eliminación de datos atípicos y en la reconstrucción de datos faltantes usando la metodología empleada por Quej et al. [25]. Posteriormente se procede a realizar un proceso de normalización, que consiste en transformar los datos de entrada de un rango amplio (original) a un rango más pequeño [26]. Esta normalización se implementa con el fin de mantener todas las variables con un mismo rango de operación, minimizar el error de regresión, mejorar la precisión y mantener la correlación entre el conjunto de datos. Su aplicación está definida por la ecuación (1) [27].

$$
X_{\text {norm }}=\frac{X_{\text {actual }}-X_{\min }}{X_{\text {max }}-X_{\min }}
$$

Donde Xnorm representa los datos normalizados, Xactual es la entrada original, Xmax y Xmin son los valores máximos y mínimos de los datos de entrada, respectivamente.

\section{Creación de la red ANFIS}

Se implementó una serie de estructuras ANFIS, considerando los tres parámetros de diseño para este tipo de redes: el número de funciones de membresía, su tipo y la estrategia de aprendizaje. El software Fuzzy Logic Toolbox TM de Matlab en su versión 9.5.0.94444 (2018b), fue la herramienta utilizada para la implementación de ANFIS, ya que proporciona funciones de línea de comandos y una interfaz completa de usuario, para el entrenamiento de sistemas de inferencia difusa de tipo Sugeno que utilizan datos específicos para el aprendizaje de entrada / salida.

Las variables de entrada seleccionadas son: temperatura, humedad, brillo solar y velocidad del viento, proporcionadas por el IDEAM en su base de datos. Adicionalmente, fue seleccionada como variable de entrada la hora de cada medición meteorológica, con el fin de evaluar si la integración de este parámetro puede llegar a optimizar los resultados de la estimación. En la figura 3 se aprecia la estructura de la red ANFIS con sus respectivas entradas y la radiación solar global como variable de salida. 
Figura 3. Estructura general de la red ANFIS

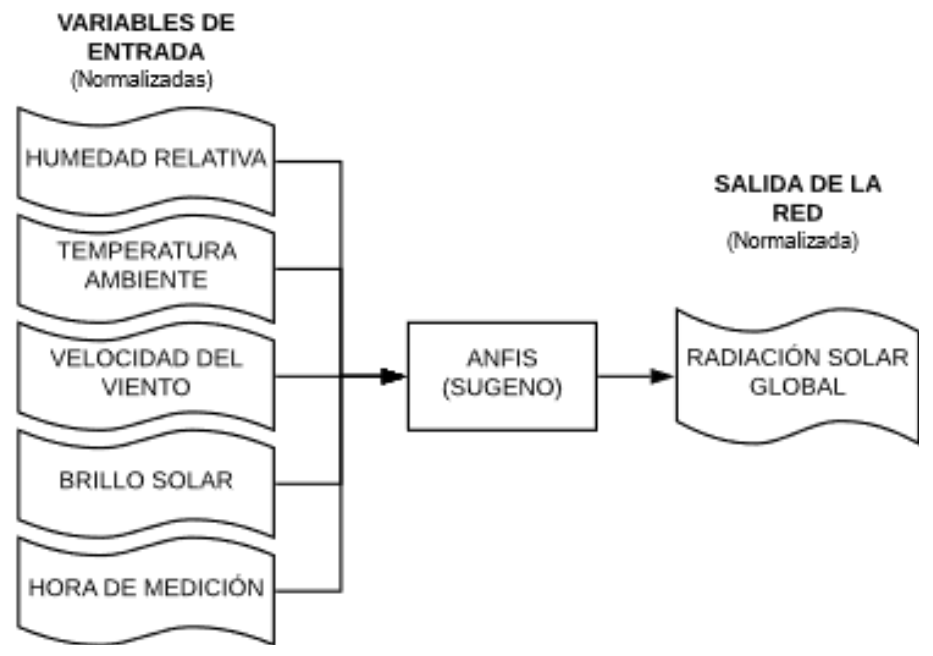

Fuente: Elaboración propia

El número de funciones de membresía en evaluación fueron 2 y 3, y los parámetros iniciales para el mapeo de las FMs se encuentran en un rango de [0,1]. Además, la red se configuró con diferentes tipos de FMs (triangular, trapezoidal, campana de Bell, campana de Gauss y campana de gauss 2 ) con el fin de identificar cuál combinación responde con el error mínimo en el entrenamiento de la red.

\section{Entrenamiento de la red.}

Después de la asignación de las FMs, se procede al entrenamiento de la red; para ello son pre-establecidas las reglas if-then de acuerdo a las posibles combinaciones de datos de entrada. El número de reglas posibles que regula la red se definen de acuerdo con la ecuación (2).

$$
\# \text { Reglas }=F M^{\mathrm{n}}
$$

Donde FM es el número de funciones de membresía y n es el número de variables de entrada. Para dos y tres funciones de membresía, se obtuvieron un total de 32 y 243 reglas que fueron incluidas respectivamente para el entrenamiento de la red. Del total de los datos, el 80 \% (18.576 de los 23.220 registros para cada variable) se utilizaron para el entrenamiento (training) y verificación (checking) de la red. Los datos de entrenamiento corresponden a los primeros ocho años disponibles (2005-2013) con frecuencia horaria.

En el entrenamiento, fueron implementadas dos estrategias de aprendizaje: una basada en el enfoque de propagación hacia atrás (back-propagation) y otra en un algoritmo híbrido derivado el cual combina el método gradiente descendiente con el estimador de mínimos cuadrados, que ofrece la Toolbox de Matlab. En inteligencia artificial, una época se refiere a una iteración en la cual todos los datos de entrenamiento son utilizados para estimar la salida y hacer un reajuste de los coeficientes del modelo. Se definió un número total de épocas con el fin de monitorizar el comportamiento del error RMSE (Root Mean Square Error) en el transcurso de las épocas y definir cuándo el error deja de disminuir significativamente. Al finalizar el entrenamiento, se escogen aleatoriamente un subgrupo pequeño de datos para verificar visualmente que el modelo de estimación efectivamente puede calcular la variable de salida a partir de los datos de entrada. 
Una vez obtenidos los resultados del entrenamiento, cada configuración de red es evaluada y comparada según los siguientes parámetros: número de épocas y valor de RMSE obtenido del proceso de entrenamiento. Aquella configuración de red que cumpla de manera óptima con estos dos indicadores es seleccionada para realizar el proceso de comprobación (testing) de los datos, cuya función de membresía y método de aprendizaje se denominará como el más apropiado para la solución del problema planteado.

\section{Ejecución y validación de la red.}

Al finalizar el entrenamiento y comparación de las diferentes topologías de la red ANFIS, se definió la red con mejor ajuste para la estimación de la radiación solar global. Seguidamente, la red es sometida a un proceso de comprobación (testing) con el $20 \%$ de los datos faltantes, es decir, con 4.644 de los 23.220 registros que corresponden a los dos años más recientes de la base de datos (2013-2015).

Este proceso de comprobación permite definir cómo se comporta la red, frente a datos totalmente desconocidos para ella; a diferencia del entrenamiento y verificación, la red sólo recibe los datos de entrada y genera una salida, sin la posibilidad de obtener una retroalimentación del error que puede presentar.

\section{Evaluación del desempeño de la red}

Para comparar los resultados de la red ANFIS con otros modelos en la literatura, se calcularon diferentes indicadores de error: RMSE, MAPE (Mean Absolute Percentage Error), y R2. Las ecuaciones (3), (4) y (5) muestran las expresiones matemáticas para cada indicador, respectivamente $[25,28,29,30]$. El valor RMSE puede ser expresado en Wh/m2 ó MJ/m2, el MAPE representa un valor porcentual (\%) y, finalmente, el R2 es un indicador adimensional del valor de correlación entre las variables estimadas y reales del proceso bajo evaluación.

$$
\begin{gathered}
M S E=\sqrt{\frac{1}{N} \sum_{i=1}^{N}\left(X_{\text {medido }}-X_{\text {estimado }}\right)^{2}} \\
A P E=\left(\frac{1}{N} \sum_{i=1}^{N}\left|\frac{X_{\text {medido }}-X_{\text {estimado }}}{X_{\text {medido }}}\right|\right) \times 100 \\
R^{2}=1-\frac{\sum_{i=1}^{N}\left(X_{\text {medido }}-X_{\text {estimado }}\right)^{2}}{\sum_{i=1}^{N}\left(X_{\text {estimado }}\right)^{2}}
\end{gathered}
$$

\section{Resultados y discusión}

Una vez explicado el proceso metodológico del entrenamiento y selección de la red ANFIS, en esta sección se presentan los resultados obtenidos del entrenamiento para cada configuración de red. Asimismo, se presenta el rendimiento de la red seleccionada y se muestra mediante gráficas la correlación que presentan las variables de entrada con respecto a la salida para evidenciar el nivel de precisión de los datos estimados con los datos reales. Por último, se realiza una comparación del error obtenido del modelo con otros trabajos reportados en la literatura. 


\section{Rendimiento y selección de la red ANFIS}

En el proceso de entrenamiento se obtienen los primeros resultados de confiabilidad de la red; Matlab permite determinar los indicadores de error cuando la red está aprendiendo, como una primera herramienta de rendimiento del modelo.

De acuerdo con los resultados obtenidos, la tabla 1 presenta un resumen de las características de entrenamiento de las redes evaluadas con 2 y 3 FMs, y dos algoritmos de aprendizaje: híbrido y propagación hacia atrás.

Tabla 1. Resultados de entrenamiento para las diferentes redes ANFIS.

\begin{tabular}{|c|c|c|c|c|}
\hline Red & Tipo de FM & No de Épocas & $\begin{array}{c}\text { Tiempo de } \\
\text { entrenamiento }\end{array}$ & RMSE \\
\hline \multicolumn{5}{|c|}{ Algoritmo de aprendizaje híbrido con 2 FMs } \\
\hline ANFIS 1 & Triangle & 66 & $18 \mathrm{~min}$ & 0.11421 \\
\hline ANFIS 2 & Gaussian & 25 & $6 \mathrm{~min}$ & 0.11327 \\
\hline ANFIS 3 & Gaussian 2 & 52 & $11 \mathrm{~min}$ & 0.11283 \\
\hline ANFIS 4 & Gbell & 50 & $14 \mathrm{~min}$ & 0.11294 \\
\hline ANFIS 5 & Trapezoidal & 30 & $9 \min$ & 0.11408 \\
\hline \multicolumn{5}{|c|}{ Algoritmo de aprendizaje de propagación hacia atrás con 2 FMs } \\
\hline ANFIS 6 & Triangle & 100 & $5 \mathrm{~min}$ & 0.13390 \\
\hline ANFIS 7 & Gaussian & 150 & $7 \mathrm{~min}$ & 0.13320 \\
\hline ANFIS 8 & Gaussian 2 & 131 & $7 \mathrm{~min}$ & 0.13335 \\
\hline ANFIS 9 & Gbell & 78 & $6 \mathrm{~min}$ & 0.13404 \\
\hline ANFIS 10 & Trapezoidal & 116 & $7 \mathrm{~min}$ & 0.12716 \\
\hline \multicolumn{5}{|c|}{ Algoritmo de aprendizaje híbrido con 3 FMs } \\
\hline ANFIS 11 & Triangle & 5 & $2 \mathrm{~h} 00 \mathrm{~min}$ & 0.11171 \\
\hline ANFIS 12 & Gaussian & 5 & 1h 57 min & 0.11056 \\
\hline ANFIS 13 & Gaussian 2 & 5 & 1h 54 min & 0.11061 \\
\hline ANFIS 14 & Gbell & 5 & $2 \mathrm{~h} 02 \mathrm{~min}$ & 0.11108 \\
\hline ANFIS 15 & Trapezoidal & 5 & $2 \mathrm{~h} 03 \mathrm{~min}$ & 0.11167 \\
\hline \multicolumn{5}{|c|}{ Algoritmo de aprendizaje de propagación hacia atrás con 3 FMs } \\
\hline ANFIS 16 & Triangle & 5 & $42 \mathrm{~min}$ & 0.31170 \\
\hline ANFIS 17 & Gaussian & 5 & $26 \mathrm{~min}$ & 0.34730 \\
\hline ANFIS 18 & Gaussian 2 & 5 & $40 \mathrm{~min}$ & 0.30054 \\
\hline ANFIS 19 & Gbell & 5 & $21 \mathrm{~min}$ & 0.30056 \\
\hline ANFIS 20 & Trapezoidal & 5 & $41 \mathrm{~min}$ & 0.30345 \\
\hline
\end{tabular}

Fuente: Elaboración propia 
El mayor número de épocas corresponde a las redes ANFIS con dos funciones de membresía y algoritmo de aprendizaje de propagación hacia atrás. El error más alto (RMSE > 0.3) ocurre para las redes ANFIS con tres funciones de membresía y aprendizaje de propagación hacia atrás. Con respecto al tiempo de entrenamiento, las redes con $2 \mathrm{FMs}$ requieren mucho menos procesamiento en comparación con las redes que tienen $3 \mathrm{FMs}$, mientras que las redes con $3 \mathrm{FMs}$ requieren solo de cinco épocas para converger. El tipo de función de membresía Gaussiana tiene el menor error en la mayoría de subgrupos de configuraciones. Según los resultados, se evidencia que el método de aprendizaje híbrido genera mejores resultados para las redes de 2 y 3 FMs que el método de aprendizaje de propagación hacia atrás.

Considerando el menor número de épocas y el menor RMSE, como se estableció en la metodología, la red de inteligencia artificial que contiene los mejores parámetros de rendimiento es ANFIS 12, con tres funciones de membresía, modelo de aprendizaje híbrido, 5 épocas, tiempo de ejecución de 1 hora con 57 minutos y un RMSE de 0.11056. Esta red es sombreada en la tabla 1 y es la escogida para el proceso de estimación de la radiación solar en la ciudad de Cúcuta.

\section{Análisis y verificación del funcionamiento de la red}

Una vez seleccionada la red definida como ANFIS 12 en la tabla 1, se realiza un proceso de verificación (checking) de los datos, para el cual se escogieron aleatoriamente 84 registros de las variables de entrada y salida con el fin de evaluar el desempeño de la red.

Los resultados de la comprobación se aprecian en la figura 4, en la cual los datos estimados siguen de cerca a los datos reales medidos, indicando que la red ANFIS seleccionada responde satisfactoriamente a la solución del problema.

Figura 4. Comparación entre los datos estimados por el modelo y los datos reales.

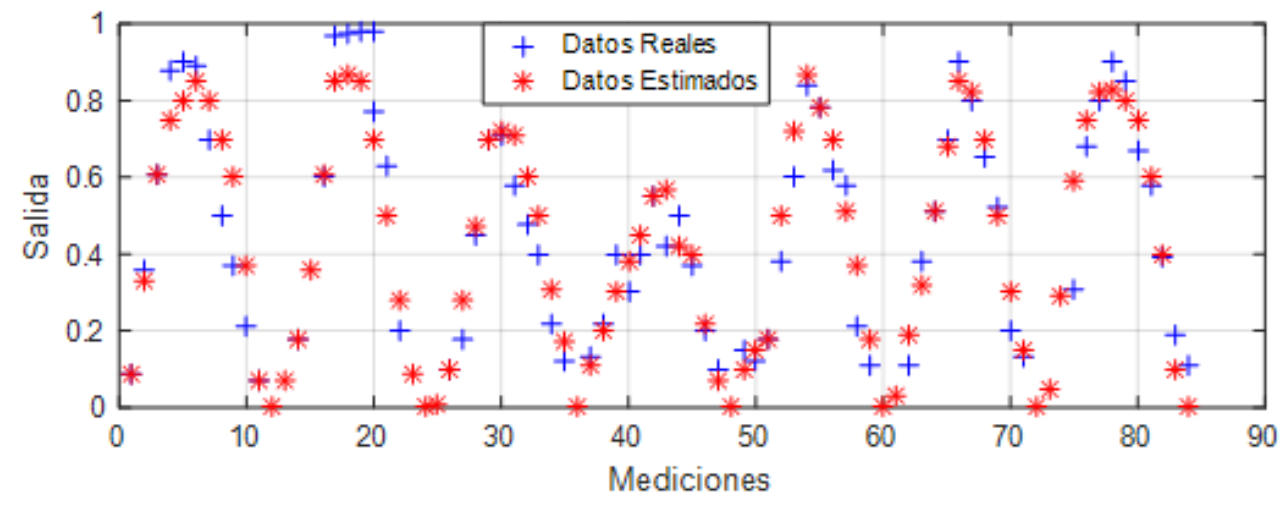

Fuente: Elaboración propia 
En la figura 5 se muestra la correlación existente entre las variables de entrada y salida del modelo obtenida a partir del Fuzzy Logic Toolbox de Matlab. La Figura 5a muestra cómo la radiación solar varía con respecto a la humedad relativa y al brillo solar. La radiación alcanza su valor máximo cuando el brillo solar es alto y la humedad relativa es nula. Para valores de humedad por encima de 0.5 , la radiación aumenta a medida que incrementa la humedad y el brillo se mantiene constante.

Figura 5. Superficie de decisión ANFIS para la estimación de la radiación solar global. (a) Humedad relativa vs Brillo solar (b) Hora vs Temperatura ambiente

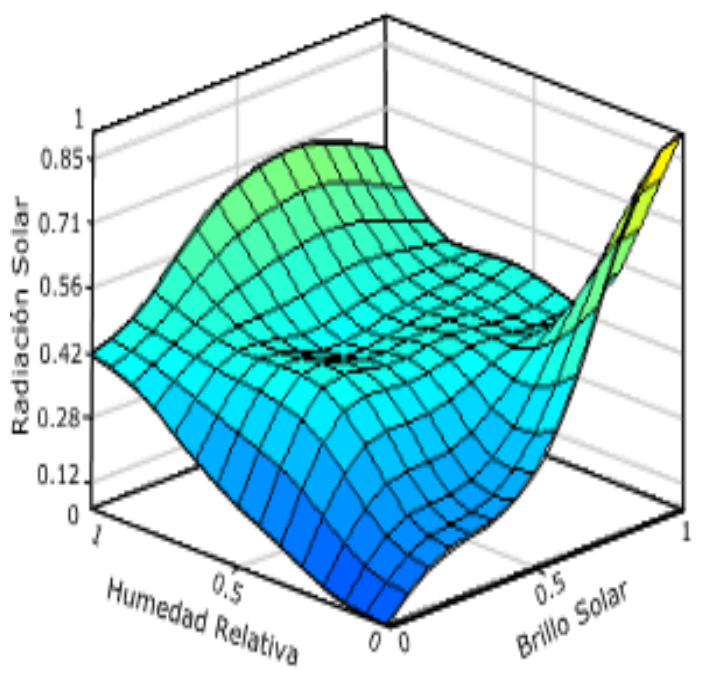

a)

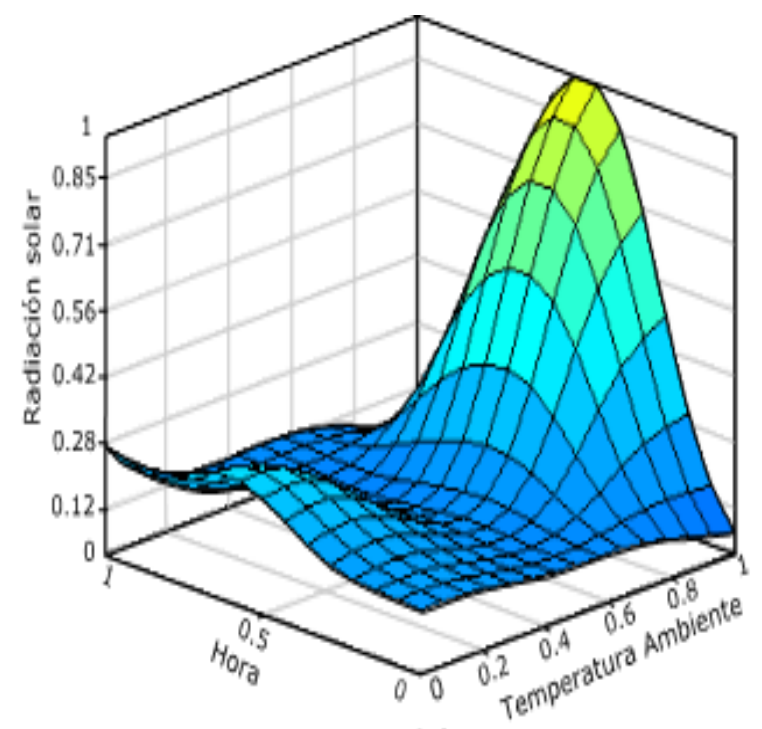

b)

Fuente: Elaboración propia

La figura $5 b$ illustra la influencia de la temperatura y la hora del día sobre la radiación solar. Como era de esperarse, a mediodía y altas temperaturas la radiación alcanza su valor máximo. También se observa que existen varios días en los cuales la temperatura es baja a mediodía, lo que se debe a días nublados o con lluvia, en los cuales los niveles de radiación son bajos. Analizando las pendientes de las curvas de superficie en las figuras $5 a$ y $5 b$, se observa que los cambios más abruptos en radiación solar ocurren cuando aumenta el brillo solar con baja humedad, y cuando se acerca al mediodía con altas temperaturas. Esto indica que la radiación solar fluctúa de acuerdo al efecto combinado de múltiples variables meteorológicas. Aunque no se muestra en la figura 5, la velocidad del viento también influye en la radiación solar; a mayor velocidad del viento, mayores niveles de radiación porque el viento ayuda a disminuir las partículas en la atmósfera aumentando la radiación solar directa. 


\section{Comprobación del desempeño de la red}

El rendimiento del modelo utilizado se calculó por medio de errores y coeficientes estadísticos. La finalidad de la comprobación (testing) es medir el desempeño de la red de estimación con valores de entrada desconocidos para ella. En la tabla 2 se muestran los resultados obtenidos en este paso.

Tabla 2. Evaluación del desempeño de la red ANFIS

\begin{tabular}{|c|c|}
\hline Indicador de error & Resultado \\
\hline MAPE (\%) & 27.8 \\
\hline RMSE (Wh/m2 / MJ/m2) & $142.23 / 0.512$ \\
\hline R2 & 0.9115 \\
\hline
\end{tabular}

Fuente: Elaboración propia

De acuerdo con Çelik, Teke y Yildirim [31], en aplicaciones prácticas, un MAPE entre $20 \%$ y $50 \%$ corresponde a una predicción razonable. Teniendo en cuenta lo anterior, el modelo ANFIS propuesto realiza estimaciones de la radiación solar a partir de datos de entrada externos con una predicción adecuada.

Ahora, el coeficiente de determinación (R2) representa el porcentaje de datos más cercano a la línea de mejor ajuste del conjunto de datos general [28]. Con lo cual, en términos de este parámetro, los resultados indican que el 91 \% de los valores de radiación solar global pronosticados están muy cerca de los valores medidos según el modelo ANFIS elegido.

El RMSE es una métrica básica ampliamente utilizada para evaluar el desempeño de los modelos de predicción [25]. Los valores más pequeños de RMSE implican una aproximación más cercana de los valores medidos por los modelos. Según lo mencionado anteriormente, con un RMSE $=142.23 \mathrm{Wh} / \mathrm{m} 2$ y R2 $=0.9115$, el modelo de inteligencia artificial cumple con los parámetros para considerarse como un buen modelo para replicar.

La figura 6 muestra una comparación de los datos medidos y estimados para la ciudad de Cúcuta. Se escogió un día de manera aleatoria para los siguientes meses: mayo, agosto, noviembre y diciembre, de los años 2014 y 2015. Como no existen estaciones en Colombia, estos meses se seleccionaron tratando de incluir diferentes condiciones climáticas durante el año. 
Figura 6. Datos Medidos vs. Estimados para el modelo de estimación por hora: a) mayo 17, 2015. b) agosto 22, 2014. c) noviembre 16, 2014. d) junio 10, 2015

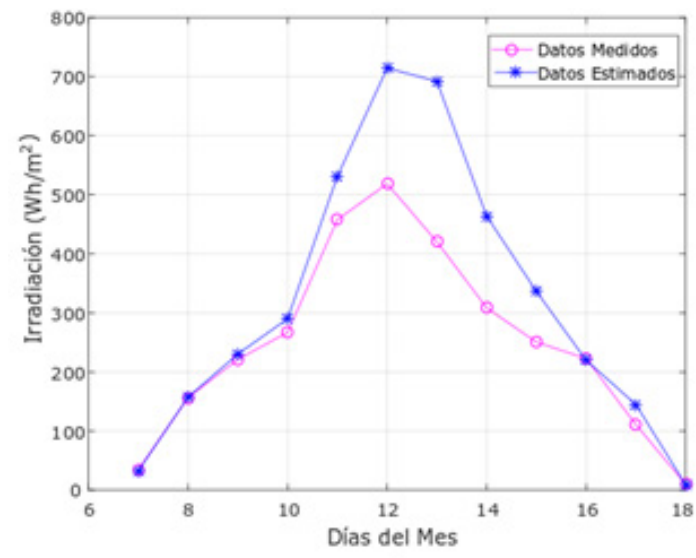

a)

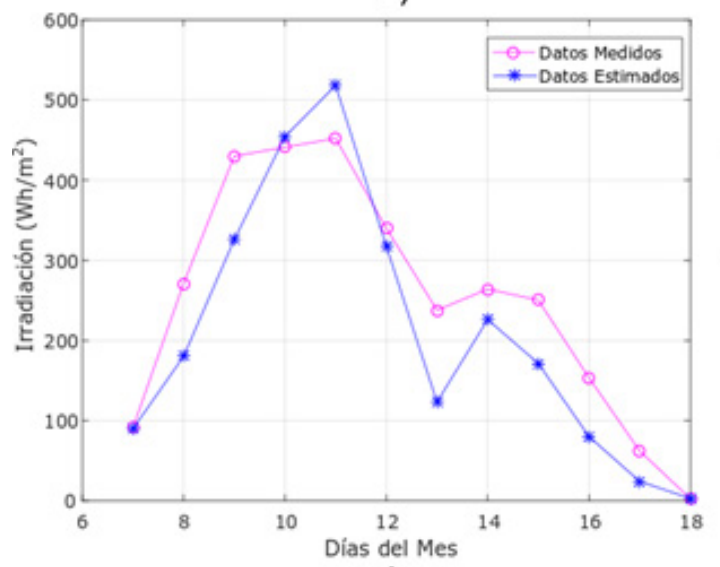

c)

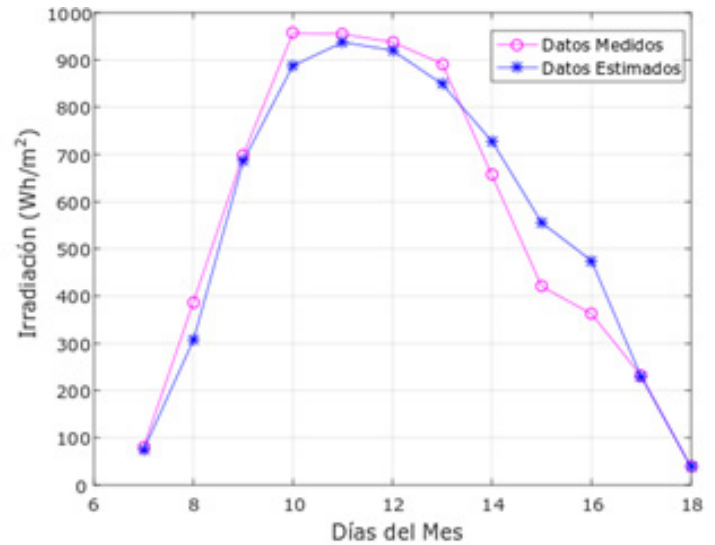

b)

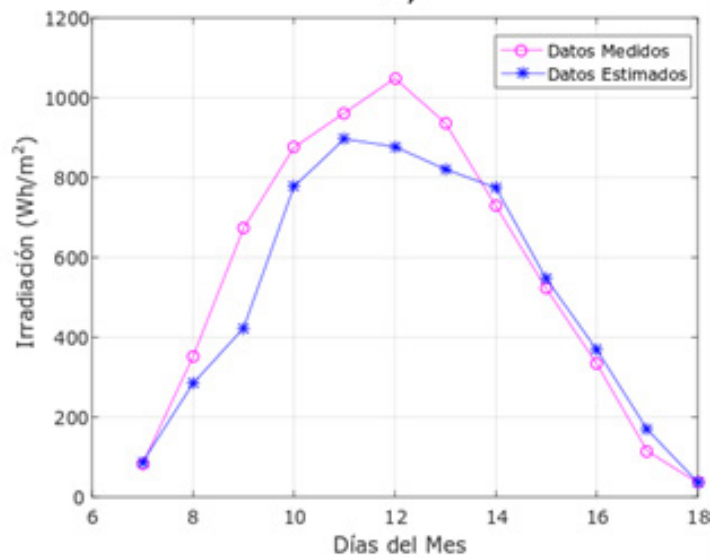

d)

\section{Fuente: Elaboración propia}

Una comparación similar a la expuesta en la figura 6 para la estimación horaria, se realiza en la figura 7, para comparar los resultados de manera diaria. Se escogió el año 2015 para la extracción de los datos debido a que es el año que presenta la menor cantidad de datos faltantes con respecto a los otros años. 
Figura 7. Datos Medidos vs. Estimados para el modelo de estimación por día: a) abril, 2015. b) junio, 2015

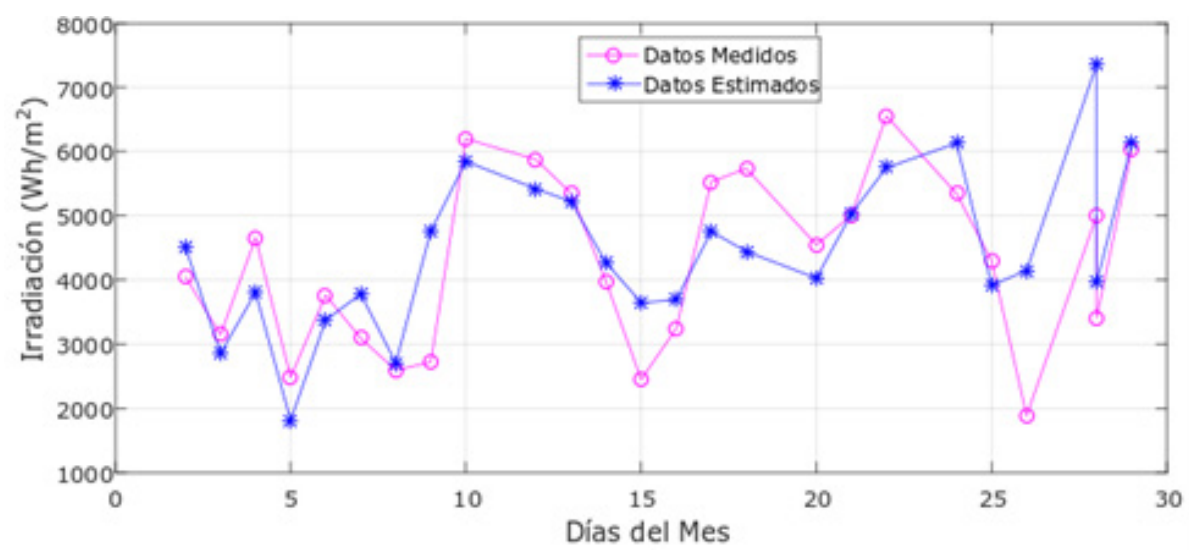

a)

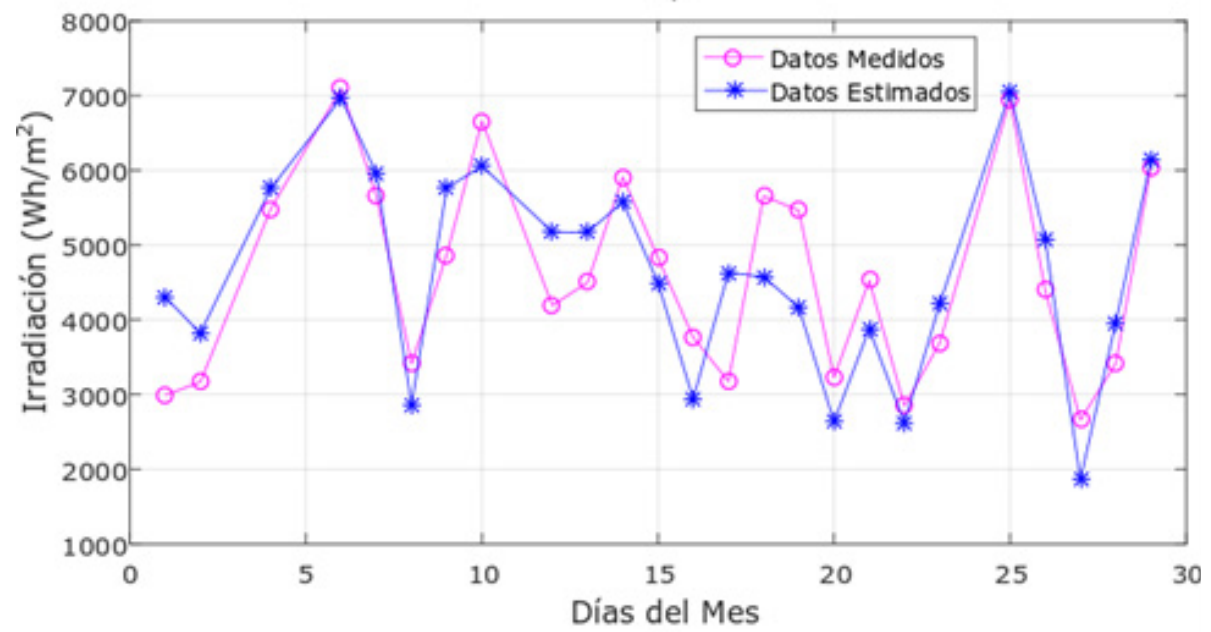

b)

Fuente: Elaboración propia

\section{Comparación de resultados}

Los resultados en términos de los indicadores de error de algunos modelos ANFIS reportados en la literatura para los diferentes rangos de estimación se presentan en la tabla 3; se destacan en azul los estudios de investigación donde el error es igual o superior a los presentados en el trabajo actual.

Cabe mencionar que usar los indicadores de error solo para seleccionar qué modelo es mejor que otro podría ser irresponsable considerando que cada modelo está entrenado con condiciones y objetivos muy diferentes. En consecuencia, la comparación en la 
tabla 3 es solo una descripción general destinada a mostrar el comportamiento de modelos similares.

\begin{tabular}{|c|c|c|}
\hline Ciudad - País & Variables de entrada & Indicadores de error \\
\hline \multirow{2}{*}{ Yucatán, México [30] } & \multirow{2}{*}{$\begin{array}{l}\text { Tmax, Tmin, radiación solar extraterrestre, días } \\
\text { lluviosos }\end{array}$} & $\mathrm{R} 2=0.645$ \\
\hline & & $\operatorname{RMSE}(\mathrm{MJ} / \mathrm{m} 2)=2.801$ \\
\hline \multirow{2}{*}{ Argelia [20] } & \multirow{2}{*}{ Latitud, longitud, altitud } & $\mathrm{R} 2=0.98$ \\
\hline & & $\operatorname{RMSE}(\mathrm{MJ} / \mathrm{m} 2)=0.0215$ \\
\hline \multirow{2}{*}{ India [32] } & \multirow{2}{*}{$\begin{array}{l}\text { Radiación solar directa, radiación solar difusa, } \\
\text { temperatura ambiente }\end{array}$} & MAPE $(\%)=2.2$ \\
\hline & & $\operatorname{RMSE}(\mathrm{MJ} / \mathrm{m} 2)=0.071$ \\
\hline \multirow[t]{2}{*}{ Iseyin, Nigeria [7] } & Tmax, Tmin, brillo solar & $\mathrm{R} 2=0.6567$ \\
\hline & & $\operatorname{RMSE}(\mathrm{MJ} / \mathrm{m} 2)=1.758$ \\
\hline \multirow{2}{*}{ Tamilnadu, India [19] } & \multirow{2}{*}{ Radiación solar global, latitud, longitud } & $\mathrm{R} 2=0.98$ \\
\hline & & $\operatorname{RMSE}(M J / m 2)=0.0078$ \\
\hline \multirow{2}{*}{ Kerman, Iran [31] } & \multirow{2}{*}{$\begin{array}{l}\text { Brillo solar, radiación solar global, radiación solar } \\
\text { extraterrestre }\end{array}$} & $\operatorname{RMSE}(\mathrm{MJ} / \mathrm{m} 2)=1.3052$ \\
\hline & & $\operatorname{MAPE}(\%)=23.052$ \\
\hline
\end{tabular}

\section{Fuente: Elaboración propia}

Los indicadores de error obtenidos del modelo ANFIS 12 propuesto, demuestran un nivel de precisión apropiado para la predicción de la radiación solar en la ciudad de Cúcuta. Estos resultados tienen relevancia porque la estación meteorológica del IDEAM carece de una gran cantidad de datos históricos, y al momento de escribir este artículo, el piranómetro no se encuentra operando. Por lo tanto, el modelo ANFIS desarrollado en este trabajo, puede utilizarse para el diseño de sistemas fotovoltaicos y para otras aplicaciones que requieran la caracterización de la radiación solar en la región.

\section{Conclusiones}

En este trabajo, se ajustó una red ANFIS variando tres parámetros: número de funciones de membresía, algoritmo de aprendizaje y tipo de función de membresía. En total se consideraron 20 configuraciones que fueron entrenadas con variables meteorológicas de entrada y la radiación solar como salida, usando el Fuzzy Logic Toolbox de Matlab. Se seleccionó el modelo con menor RMSE y se encontró que la radiación solar varía según el efecto conjunto de múltiples variables de entrada.

La red adaptativa de inferencia neuro-difusa (ANFIS) que fue propuesta en esta investigación, utiliza cinco variables meteorológicas de entrada: humedad relativa, temperatura ambiente, velocidad del viento, brillo solar y hora de la medición para estimar la radiación solar global en la ciudad de Cúcuta. Después de entrenar la red ANFIS, los resultados de comprobación (RMSE $=142 \mathrm{kWh} / \mathrm{m} 2$ y R2 $=0.9115)$ y la comparación con otros modelos ANFIS en la literatura, demuestran que el modelo tiene una precisión adecuada y que puede utilizarse para el dimensionamiento de sistemas fotovoltaicos en la región.

El menor error de la red ANFIS se obtuvo utilizando el algoritmo de aprendizaje híbrido y tres funciones de membresía. Estos resultados pueden utilizarse como punto de referencia para extrapolar el modelo a otras regiones del país donde no se cuenta con una amplia cantidad de estaciones meteorológicas en funcionamiento para la medición de radiación solar. Adicionalmente, el modelo desarrollado puede ser útil para agencias gubernamentales locales o nacionales y para el sector privado, para cuantificar el potencial solar de la ciudad, y realizar análisis tecno-económicos de sistemas fotovoltaicos. 


\section{Referencias Bibliográficas}

1. REN21, “Renewables 2020 Global Status Report,” 2020.

2. D. Henao León, A. C. Báez Alarcón, and J. B. Pedroza Rojas, “Metodología para determinar la viabilidad de generación de energía eléctrica por medio del recurso eólico," Investig. e Innovación en Ing., vol. 6, no. 2, pp. 6-15, 2018.

3. S. Sobri, S. Koohi-Kamali, and N. A. Rahim, "Solar photovoltaic generation forecasting methods: A review," Energy Convers. Manag., vol. 156, pp. 459-497, 2018.

4. Instituto de Hidrología Meteorología y Estudios Ambientales (IDEAM), “Atlas de Radiación Solar, Ultravioleta y Ozono de Colombia," 2015. [Online]. Available: http://atlas.ideam.gov.co/ visorAtlasRadiacion.html.

5. W. Contreras, M. G. Galban, and S. B. Sepúlveda, "Análisis estadístico de la radiación solar en la ciudad de Cúcuta," Entre Cienc. e Ing., vol. 12, no. 23, p. 16, 2018.

6. E. Noriega-Angarita, V. Sousa-Santos, M. Quintero-Duran, and C. Gil-Arrieta, "Solar radiation prediction for dimensioning photovoltaic systems using artificial neural networks," Int. J. Eng. Technol., vol. 8, no. 4, pp. 1817-1825, 2016.

7. L. Olatomiwa, S. Mekhilef, S. Shamshirband, and D. Petković, “Adaptive neuro-fuzzy approach for solar radiation prediction in Nigeria," Renew. Sustain. Energy Rev., vol. 51, pp. 1784-1791, 2015.

8. R. Kumar, R. K. Aggarwal, and J. D. Sharma, "Comparison of regression and artificial neural network models for estimation of global solar radiations," Renew. Sustain. Energy Rev., vol. 52, pp. 1294-1299, 2015.

9. J. I. Roa Mora, “Análisis de las Alternativas del Diseño de un Sistema Difuso para la Predicción del Precio del Bitcoin (BTC)," Investig. e Innovación en Ing., vol. 7, no. 1, pp. 34-46, 2019.

10. K. Mohammadi, S. Shamshirband, A. Kamsin, P. C. Lai, and Z. Mansor, "Identifying the most significant input parameters for predicting global solar radiation using an ANFIS selection procedure," Renew. Sustain. Energy Rev., vol. 63, pp. 423-434, 2016.

11. L. M. Halabi, S. Mekhilef, and M. Hossain, "Performance evaluation of hybrid adaptive neuro-fuzzy inference system models for predicting monthly global solar radiation," Appl. Energy, vol. 213, pp. 247-261, 2018.

12. D. Jain and M. Lalwani, "Prediction of irradiation: A comparative study of ANFIS," International Conference on Circuit, Power and Computing Technologies (ICCPCT), 2017, pp. 1-6.

13. A. Khosravi, R. N. N. Koury, L. Machado, and J. J. G. Pabon, "Prediction of hourly solar radiation in Abu Musa Island using machine learning algorithms," J. Clean. Prod., vol. 176, pp. 63-75, 2018.

14. K. Mohammadi, S. Shamshirband, C. W. Tong, K. A. Alam, and D. Petković, "Potential of adaptive neurofuzzy system for prediction of daily global solar radiation by day of the year," Energy Convers. Manag., vol. 93, pp. 406-413, 2015.

15. A. Mohan, P. Kuchhal, and M. G. Sharma, "Prediction Models for Global Solar Radiations, Diffused Radiations and Direct Solar Radiations Using ANFIS," International Conference on Intelligent Communication, Control and Devices, 2017, pp. 27-37.

16. A. Muhammad, M. S. Gaya, RAliyu, R.A. Abdulkadir, I.D. Umar, L. A. Yusuf, et al., "Forecasting of global solar radiation using ANFIS and ARMAX techniques," IOP Conference Series: Materials Science and Engineering, 2018, vol. 303, pp. 1-6. 
17. L. Zou, L. Wang, L. Xia, A. Lin, B. Hu, and H. Zhu, "Prediction and comparison of solar radiation using improved empirical models and Adaptive Neuro-Fuzzy Inference Systems," Renew. Energy, vol. 106, pp. 343-353, 2017.

18. S. Mohanty, "ANFIS based Prediction of Monthly Average Global Solar Radiation over Bhubaneswar (State of Odisha)," Int. J. Ethics Eng. Manag. Educ., vol. 1, no. 5, pp. 97-101, 2014.

19. T. R. Sumithira and A. Nirmal Kumar, "Prediction of monthly global solar radiation using adaptive neuro fuzzy inference system (ANFIS) technique over the state of Tamilnadu (India): A comparative study," Appl. Sol. Energy, vol. 48, pp. 140-145, 2012.

20. A. Mellit, S. A. Kalogirou, S. Shaari, H. Salhi, and A. Hadj Arab, "Methodology for predicting sequences of mean monthly clearness index and daily solar radiation data in remote areas: Application for sizing a stand-alone PV system," Renew. Energy, vol. 33, no. 7, pp. 1570-1590, 2008.

21. J. S. R. Jang, "ANFIS: Adaptive-Network-Based Fuzzy Inference System," IEEE Trans. Syst. Man Cybern., vol. 23, no. 3, pp. 665-685, 1993.

22. A. M. Abdulshahed, A. P. Longstaff, and S. Fletcher, "The application of ANFIS prediction models for thermal error compensation on CNC machine tools," Appl. Soft Comput., vol. 27, pp. 158-168, 2015.

23. A. Abraham, "Adaptation of Fuzzy Inference System Using Neural Learning," Fuzzy Systems Engineering, vol. 181, N. Nedjah and L. Macedo Mourelle, Eds. Springer, Berlin, Heidelberg, 2005, pp. 53-83.

24. Instituto de Hidrología Meteorología y Estudios Ambientales de Colombia (IDEAM), "Solicitud de Información," 2014. [Online]. Available: http://www.ideam.gov.co/solicitud-de-informacion.

25. V. H. Quej, J. Almorox, J. A. Arnaldo, and L. Saito, "ANFIS, SVM and ANN soft-computing techniques to estimate daily global solar radiation in a warm sub-humid environment," J. Atmos. Solar-Terrestrial Phys., vol. 155, pp. 62-70, 2017.

26. H. B. Yıldııım, Ö. Çelik, A. Teke, and B. Barutçu, "Estimating daily Global solar radiation with graphical user interface in Eastern Mediterranean region of Turkey," Renew. Sustain. Energy Rev., vol. 82, pp. 1528-1537, 2018.

27. U.K. Das, K. Soon Tey, M. Seyedmahmoudian, S. Mekhilef, M.Y. Idna Idris, W.V. Deventer, et al., "Forecasting of photovoltaic power generation and model optimization: A review," Renew. Sustain. Energy Rev., vol. 81, pp. 912-928, 2018.

28. N. Premalatha and A. Valan Arasu, "Prediction of solar radiation for solar systems by using ANN models with different back propagation algorithms," J. Appl. Res. Technol., vol. 14, no. 3, pp. 206-214, 2016.

29. K. Chiteka and C. C. Enweremadu, "Prediction of global horizontal solar irradiance in Zimbabwe using artificial neural networks," J. Clean. Prod., vol. 135, pp. 701-711, 2016.

30. A. Qazi, H. Fayaz, A. Wadi, R. G. Raj, N. A. Rahim, and W. A. Khan, "The artificial neural network for solar radiation prediction and designing solar systems: A systematic literature review," J. Clean. Prod., vol. 104, pp. 1-12, 2015.

31. Ö. Çelik, A. Teke, and H. B. Yildirim, "The optimized artificial neural network model with LevenbergMarquardt algorithm for global solar radiation estimation in Eastern Mediterranean Region of Turkey," J. Clean. Prod., vol. 116, pp. 1-12, 2016.

32. H. K. Yadav, Y. Pal, and M. M. Tripathi, "Short-Term PV Power Forecasting Using Adaptive Neuro-Fuzzy Inference System," IEEE Power India International Conference, (PIICON), 2018, pp. 1-6. 